\title{
Myosin heavy chain isoforms expression, calpain system and quality characteristics of different muscles in goats
}

\begin{abstract}
Myosin heavy chain (MHC) isoforms in goat muscles and their possible relationships with meat quality have not been fully elucidated. This study characterized the MHC isoforms in different caprine muscles using sodium dodecyl sulphate glycerol gel electrophoresis (SDSGGE). The relationships between MHC isoforms, calpain systems and meat quality characteristics of different muscles in goats were examined. Four muscles, namely infraspinatus (IF), longissimus dorsi (LD), psoas major (PM) and supraspinatus (SS) were obtained from ten Boer crossbred bucks (7-10 months old; $26.5 \pm 3.5 \mathrm{~kg}$, BW). The percentages of MHC I, MHC IIa and MHC IIx in SS, IF, PM and LD were 47.2, 38.3, 32.1, 11.9; 28.0, 42.1, 33.0, 36.4; and 24.8, 19.6, 34.9 and 51.7, respectively. IF and SS had higher levels of calpastatin, total collagen and insoluble collagen contents than did PM and LD. PM had longer sarcomere length than did other muscles. LD had higher collagen solubility, troponin-T degradation products and glycogen content than did other muscles. These results infer that variable fiber-type composition could account partially for the differences in the physicochemical properties of goat muscles.
\end{abstract}

Keyword: Calpastatin; Collagen; Fiber; Glycogen; Sarcomere; SDS-GGE; Troponin T 